\title{
A Replication of "Functional Equivalence of Verbal and Spatial Information in Serial Short-Term Memory (1995; Experiments 2 and 3)"
}

\author{
Dominic Guitard a, Jean Saint-Aubin
}

a École de psychologie, Université de Moncton

Abstract $\square$ The present study is a direct replication of Experiments 2 and 3 of Jones, Farrand, Stuart, \& Morris (1995). Functional Equivalence of Verbal and Spatial Information in Serial Short-Term Memory. Journal of Experimental Psychology: Learning, Memory, and Cognition, 21, 1008-1018.

Keywords - Short-term memory, Functional equivalence, Replication study

Đj jean.saint-aubin@umoncton.ca

\section{Introduction}

In memory research in general, and short-term memory in particular, there is a vigorous debate about modularity (see, e.g., Surprenant \& Neath, 2009). Modularity is at the heart of the working memory model in which verbal and visual-spatial information would be maintained through distinct memory subsystems (Baddeley, 1990). In support of this view, dissociations were found between short-term recall of verbal and visual-spatial information (e.g., Farmer, Berman, \& Fletcher, 1986). In 1995, Jones Farrand, Stuard and Morris challenged the modularity of working memory by demonstrating the functional equivalence of immediate memory for verbal and visual-spatial information. More specifically, Jones et al. very elegantly equated the verbal and the spatial memory tasks. In the verbal condition, participants performed an order reconstruction task. Seven letters were sequentially presented in the center of the screen. At recall, all letters reappeared simultaneously in a random order in a row. Participants were asked to click on them in their presentation order. In the spatial condition, seven dots were sequentially presented at various locations on the screen and at recall, all dots reappeared simultaneously. Participants responded by clicking on them in their presentation order. In addition, Jones et al. introduced a verbal and a spatial interfering task: articulatory suppression and spatial tapping, respectively. In their paper, cited 150 times according to Web of Science, the key finding is that tapping impaired verbal and spatial order reconstruction tasks to the same extent (Experiment 2) and similarly, articulatory suppression had the same detrimental effect on verbal and spatial order reconstruction tasks (Experiment 3).

There have been two conceptual replications of Jones' et al. (1995) study (Guérard \& Tremblay, 2008; Meiser \& Klauer, 1999). In both replications, the detrimental effect of tapping on verbal and spatial memory tasks was observed. However, contrary to the results of Jones et al., the detrimental effect of tapping on the verbal memory task was significantly smaller than its detrimental effect on the spatial task. Even more problematic, both replications failed to find a significant detrimental effect of articulatory suppression on the spatial memory task.

The diverging findings between the original study and the two conceptual replications can either be due to methodological differences across studies or to none replicability of the original findings. For instance, Meiser and Klauer (1999) used Corsi blocks for the spatial reconstruction task instead of dots. With Corsi blocks, the same items are used across trials. In other words, a close pool of items is used, while Jones et al. (1995) used an open pool of items with different dot locations at each trial. Similarly, Guérard and Tremblay (2008) used a closed set of items with the same seven dot locations for all trials. The set size difference between Jones' et al. study and the two replications may account for the divergent pattern of results. In effect, previous studies have shown that a number of benchmark working memory effects are modulated by the size of the memory set (see, e.g., Roodenrys \& 

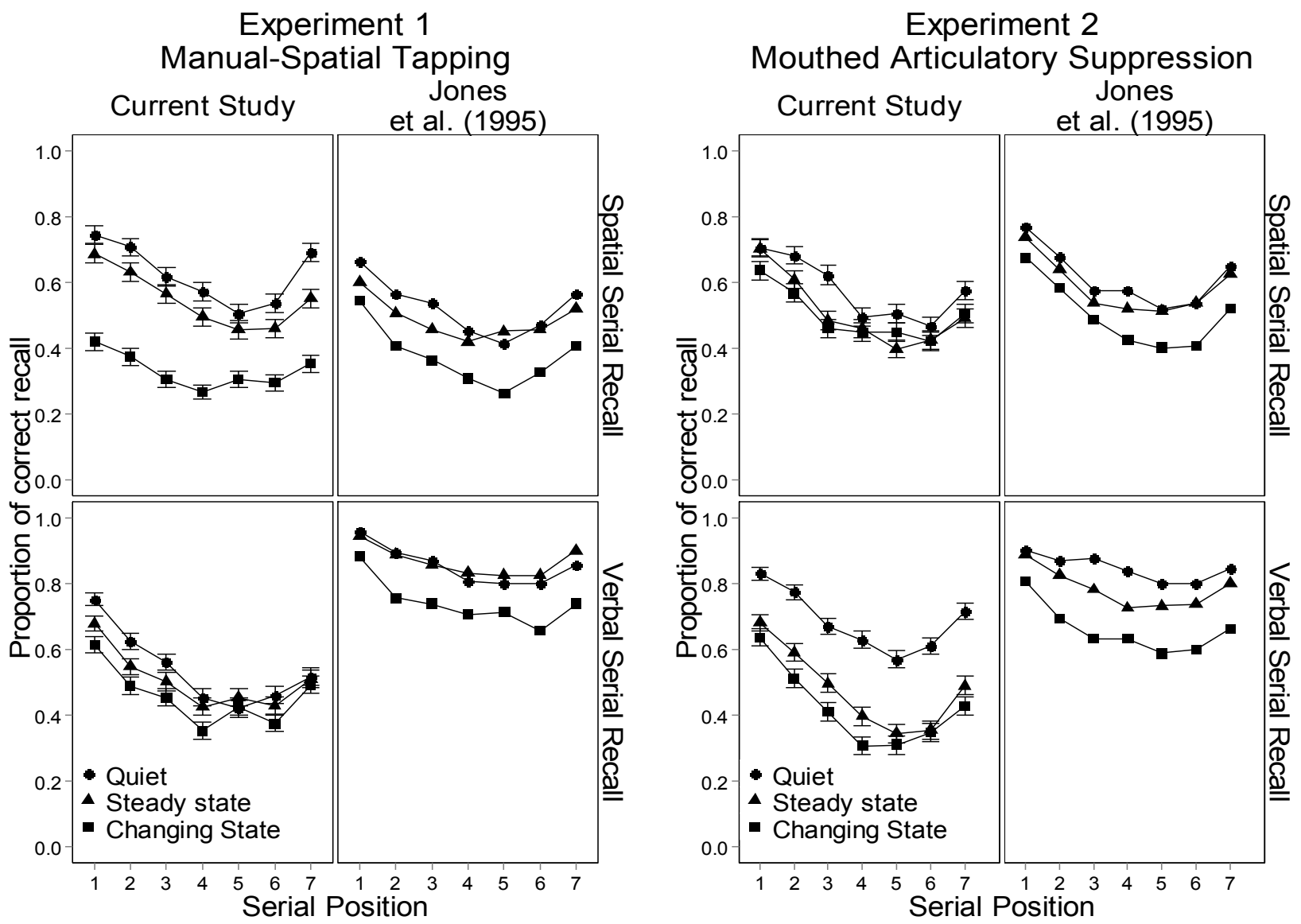

Figure 1 - Proportion of correct recall as a function of experiment, memory task, interference, and serial position. Error bars represent 95\% within-participant confidence intervals computed according to Morey's (2008) procedure.

Quinlan, 2000). In the present study, we replicated the second and third experiments of Jones et al.

\section{General Method}

\section{Participants}

In each experiment, 36 students (18 in the verbal recall task and 18 in the spatial recall task) from Université de Moncton volunteered to participate in this experiment. All reported speaking French at home, and normal or corrected to normal vision. No participant took part in both experiments.

\section{Materials and Procedure}

The materials and procedure were the same as those used by Jones et al. (1995) except for the following changes. The experiment was controlled with E-Prime 2.0 and the stimuli were presented on a $47.72 \mathrm{~cm}(18$ inches) screen. The experiment was run in French, instead of English. Finally, in Experiment 1, tapping was executed by pressing the 11 the outside keys of the numeric keypad, instead of the 12 outside keys of the Macintosh LC II computers used in the original study.

\section{Experiment 1 and 2}

In Experiment 1, we replicated Experiment 2 of Jones et al. (1995) in which they used manual spatial tapping as their interfering task and in Experiment 2, we replicated Experiment 3 of Jones et al. in which they used articulatory suppression as their interference task.

\section{Results}

As shown in Figure 1 and Table 1, in both experiments, there was a large and significant interaction between domain (verbal vs. spatial) and interference. This interaction reflects the larger detrimental effect of the interference task when it is in the same domain as the memory task (articulatory suppression for the verbal task and tapping for the dot task) than when it is the other domain (articulatory suppression for the dot task 
Table 1 - ANOVAs from Jones et al. (1995) and the present study

\begin{tabular}{|c|c|c|c|c|c|c|}
\hline \multirow[b]{2}{*}{ Source } & \multicolumn{3}{|c|}{ Original study } & \multicolumn{3}{|c|}{ Replication } \\
\hline & $F$ & $p$ & $\eta^{2} \mathrm{p}$ & $F$ & $p$ & $\eta^{2} \mathrm{p}$ \\
\hline \multicolumn{7}{|c|}{ Experiment 1 (manual spatial-tapping) } \\
\hline Domain $(D)$ & 65.53 & .001 & .65 & 8.89 & .01 & .21 \\
\hline Interference $(I)$ & 17.03 & .001 & .33 & 54.02 & .001 & .61 \\
\hline Serial Position $(S P)$ & & & & 58.90 & .001 & .63 \\
\hline$D \times I$ & .59 & .60 & .02 & 17.75 & .001 & .34 \\
\hline Interference for verbal & & & & 6.01 & .01 & .26 \\
\hline Interference for spatial & & & & 57.73 & .001 & .77 \\
\hline$D \times S P$ & & & & 2.29 & .05 & .06 \\
\hline$I \times S P$ & & & & 1.93 & .05 & .05 \\
\hline$D \times I \times S P$ & .56 & .87 & .02 & .87 & .57 & .03 \\
\hline \multicolumn{7}{|c|}{ Experiment 2 (articulatory suppression) } \\
\hline Domain $(D)$ & 14.74 & .001 & .30 & 3.39 & .07 & .09 \\
\hline Interference $(I)$ & 15.22 & .001 & .31 & 48.40 & .001 & .59 \\
\hline Serial Position $(S P)$ & & & & 71.33 & .001 & .68 \\
\hline$D \times I$ & .99 & .38 & .03 & 13.36 & .001 & .28 \\
\hline Interference for verbal & & & & 48.23 & .001 & .74 \\
\hline Interference for spatial & & & & 6.59 & .01 & .28 \\
\hline$D \times S P$ & & & & 1.17 & .32 & .03 \\
\hline$I \times S P$ & & & & 1.24 & .25 & .04 \\
\hline$D \times I \times S P$ & 1.22 & .27 & .03 & 1.21 & .27 & .03 \\
\hline
\end{tabular}

Note: Blank cells are due to missing information in the original article.

and tapping for the verbal task).

For sake of comprehensiveness, a further analysis was computed. In addition to the functional equivalence of verbal and spatial immediate memory tasks, Jones et al. (1995) showed that changing-state interference was significantly more detrimental than steady-state, which did not induce a significant memory decrement compared to the control quiet condition. Here, post hoc comparisons (Tukey's honestly significant difference) revealed that in Experiment 1, all three conditions (quiet, steady and changing state) significantly differ one from the other. In Experiment 2, with articulatory suppression, a different pattern emerged. In effect, both interference conditions induced a significantly lower recall performance than the control quiet condition, but there was no significant difference between changing and steady state conditions.

\section{General Discussion}

In the present study, we partially replicated the main findings of Jones et al. (1995). First, as observed by Jones et al., qualitatively in Experiment 1, changing state tapping was more detrimental than steady-state. However, in Experiment 2 with articulatory suppression, the difference between changing and steady state was small and did not reach significance. Second, as in the original study, there was a significant detrimental effect of each secondary task on the memory task from the other domain (articulatory suppression on the dot task and tapping on the verbal memory task). However, contrary to Jones et al (1995), the interference effect was much larger when the secondary and the main tasks were in the same domain than when they were in different domains. The difference in effect sizes across studies was very large 
with the interaction accounting for only 2 and 3 percent of variance in Jones et al., while here it accounted for 34 and 28 percent of variance. Our interaction nicely reproduced the results reported by Meiser and Klauer (1999) and Guéard and Tremblay (2008). For instance, in Guérard and Tremblay's study, the value of the partial eta squared for the interaction was of $18 \%$ and $69 \%$ for the tapping and suppression condition respectively.

In summary, our results confirm the presence of interference across domains, but show that this interference is of smaller magnitude than withindomain interference. Our results further show the risk of arguing for the null effect-as it was the case of Jones et al. (1995) with their non-significant interaction-in the context of null hypothesis testing.

\section{Authors' notes and acknowledgments}

This research was supported by a discovery grant from the Natural Science and Engineering Research Council of Canada to Jean Saint-Aubin. We thank Marie-Ève Saint-Louis for programming the experiments. Correspondence concerning this article should be addressed to Jean Saint-Aubin, École de psychologie, Université de Moncton, Moncton, New Brunswick, E1A 3E9, Canada.

\section{References}

Baddeley, A. D. (1990). Human memory: Theory and practice. Needham Heights, MA: Allyn \& Bacon.

Farmer, E. W., Berman, J. V., \& Fletcher, Y. L. (1986).
Evidence for a visuo-spatial scratch-pad in working memory. The Quarterly Journal of Experimental Psychology A: Human Experimental Psychology, 38, 675-688. doi:10.1080/14640748608401620

Guérard, K., \& Tremblay, S. (2008). Revisiting evidence for modularity and functional equivalence across verbal and spatial domains in memory. Journal of Experimental Psychology: Learning, Memory, and Cognition, 34, 556-569. doi:10.1037/02787393.34.3.556

Jones, D., Farrand, P., Stuart, G., \& Morris, N. (1995). Functional equivalence of verbal and spatial information in serial short-term memory. Journal of Experimental Psychology: Learning, Memory, and Cognition, 21, 1008-1018. doi:10.1037/02787393.21.4.1008

Meiser, T., \& Klauer, K. C. (1999). Working memory and changing-state hypothesis. Journal of Experimental Psychology: Learning, Memory, and Cognition, 25, 1272-1299. doi:10.1037/0278-7393.25.5.1272

Morey, R. D. (2008). Confidence Intervals from Normalized Data: A correction to Cousineau (2005). Tutorial in Quantitative Methods for Psychology, 4, 61-64.

Roodenrys, S., \& Quinlan, P. T. (2000). The effects of stimulus set size and word frequency on verbal serial recall. Memory, 8 , 71-78. doi:10.1080/096582100387623

Surprenant, A. M., \& Neath, I. (2009). Principles of memory. New York, NY, US: Psychology Press.

\section{Citation}

Guitard, D., \& Saint-Aubin, J. (2015). A Replication of "Functional Equivalence of Verbal and Spatial Information in Serial Short-Term Memory (1995; Experiments 2 and 3)". The Quantitative Methods for Psychology, 11 (2), r4-r7. 